\title{
Application des algorithmes d'inversion de la profondeur à des données de terrain
}

\author{
Marie-Aude Pradal ${ }^{1,}$,, Stéphan Grilli ${ }^{1}$, John Dugan ${ }^{2}$, Zandy Williams ${ }^{2}$
}
1. Department of Ocean Engineering University of Rhode Island, Narragansett, RI 02882, U.S.A. $\quad$ fax. : 001-401-874-6837.; email:ma_pradal@hotmail.com grilli@oce.uri.edu

2. Areté Associates, 1725 Jefferson Davis Highway Suite 703 Arlington, Virginia 22202, U.S.A. tel.: 001-703-413-0290 email:dugan@arete-dc.com williams@arete-dc.com

\section{Résumé}

Les méthodes d'inversion de profondeur estiment la bathymétrie en se basant sur des observations par télédétection de vagues de surface en cours de shoaling. Les vitesses de phase sont déduites de ces mesures. La profondeur est calculée en inversant une relation de dispersion. L'utilisation de données de terrain de luminance de la surface océanique aboutit aux mêmes conclusions que lors des simulations en canal à houle numérique. La profondeur calculée par la théorie linéaire est surestimée du fait de l'omission de la dispersion d'amplitude. L'algorithme non-linéaire (Grilli, 1998) donne de meilleurs résultats, comme le montre la comparaison des profondeurs mesurées lors de la campagne SHOWEX à celles calculées par les théories linéaire et non-linéaire.

\begin{abstract}
Depth inversion consists in estimating the ocean bottom topography based on properties of shoaling waves observed on the ocean surface, using remote sensing methods. Wave phase speed is typically calculated from these measurements and depth is retrieved from inverting a wave dispersion relationship. Results based on the analysis of field measurements of brightness variations show key features similar to those calculated in numerical wave tanks. Linear wave theory overestimates depth in shallow water. This is due to nonlinear effects leading to amplitude dispersion. A nonlinear theory (Grilli,1998) provides more accurate results when comparing to depth measurements obtained during SHOWEX.
\end{abstract}

\footnotetext{
${ }^{a}$ Nouvelle adresse : Université de la Méditerranée Centre d'Océanologie de Marseille. Campus de Luminy, 163 av de Luminy Case 90113288 Marseille Cedex 9. tel : 0491829110
} 


\section{VII ${ }^{\grave{e} m e s}$ Journées Nationales Génie Civil-Génie Côtier, Anglet, France, 15-17 Mai 2002}

\section{$\underline{\text { 1. Introduction }}$}

La connaissance de la bathymétrie est primordiale pour de nombreux problèmes d'ingénierie côtière. L'accès direct à cette donnée par des méthodes de sondage sonar présente cependant des limites en terme de durée des campagnes et de commodité des mesures. De ce fait des méthodes indirectes ont été développées, dites d'inversion de la profondeur, qui consistent à estimer la bathymétrie en se basant sur des observations de télédétection des vagues de surface au cours du shoaling. Ces observations sont combinées à des équations prédisant les propriétés de ces vagues, telles que leur vitesse de phase, qui varient avec la profondeur d'eau. L'inversion de ces équations permet de prédire la profondeur. Comme les vagues proches de la côte deviennent fortement non-linéaires, les équations utilisées doivent prendre ces non-linéarités en compte durant le shoaling. Pour ce faire, un modèle numérique complètement non-linéaire est utilisé pour simuler le shoaling d'un grand nombre de vagues, couvrant le domaine paramétrique d'intérêt (pour plus de détails voirGrilli et Horrillo, 1997 et Grilli, 1998). Ce travail présente l'application de ces méthodes d'inversion de la profondeur à des données de terrain récemment obtenue à Duck, N.C., U.S.A durant l'expérience SHOWEX (Dugan et al, 2001).

La seconde partie de cette article définira plus précisément quel est la problématique de cette étude, puis la troisième partie, présentera le principe de l'inversion de la profondeur, la quatrième partie introduira les données de terrain utilisées, et le principe de leur traitement. Enfin, la cinquième partie montrera comment notre algorithme d'inversion permet de calculer la bathymétrie et présentera les résultats des calculs, avant de conclure.

\section{Problématique de ce travail}

La profondeur d'eau dans la direction normale $h(x)$ au rivage, peut être estimée en inversant une relation dite de dispersion de houle, qui exprime la célérité (ou vitesse de phase) $c$ de la houle, en fonction de $h$. La théorie de houle linéaire énonce cette relation de la manière suivante,

$$
c=c_{0} \tanh (k h)
$$

(où $k=2 \pi / L$ et $c_{0}=g T /(2 \pi) ; L:$ longueur d'onde ; $T$ : période) qui donne de bonnes prédictions en cas de faible non-linéarité, c'est-à-dire dans la zone qui s'étend des grandes profondeurs jusqu'à une certaine distance du rivage, avant que la hauteur $H$ (et cambrure $H / L$ ou $k H$ ) des vagues n'augmente significativement en fin de shoaling. A ce moment-là, à la dispersion de fréquence décrite par (1), s'ajoute la dispersion d'amplitude, qui n'apparaît formellement dans la théorie que si on la pousse au moins jusqu'au 3ème ordre de non-linéarité. Selon celle-ci, les vagues sont d'autant plus rapides qu'elles sont cambrées. En conséquence, l'utilisation de la théorie linéaire donne des célérités sous-estimées, et donc des profondeurs surestimées (Grilli, 1998). 


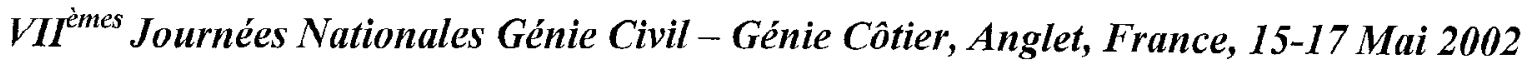

Les équations d'ondes longues non-linéaires (au premier ordre), quant à elles, expriment la vitesse de phase des vagues déferlantes uniquement en fonction de leur amplitude et de la profondeur,

$$
c=g(H+h)^{1 / 2}
$$

Dugan and Williams (2000) utilisent (2) pour prédire la profondeur au dela du point de déferlement, en exprimant la hauteur des vagues à l'aide d'un critère de déferlement glissant, $H=\gamma h$ (où $\gamma \approx 0.65$ est le coefficient de déferlement). Utilisant des mesures de célérités basées sur des images aériennes suivant les trajectoires des déferlantes, ils montrent que leurs prédictions de profondeur sont raisonnables, comparées aux données de terrain. Donc, corrélativement, Eq. (2) représente correctement la célérité des vagues déferlant de cette manière.

Dans la zone de fin de shoaling, qui constitue le centre d'intérêt de notre étude, il apparaît que nous devions utiliser une équation de dispersion tenant compte des phénomènes dispersif de fréquence et non-linéaire : la célérité s'exprime alors en fonction de la profondeur relative $k h$ et de la cambrure $k H$. Cette équation a été développée, pour des vagues périodiques en shoaling sur des pentes faibles, par Grilli (1998), sur la base d'un modèle numérique potentiel complètement nonlinéaire. Un résumé de ces résultats est donné ci-dessous.

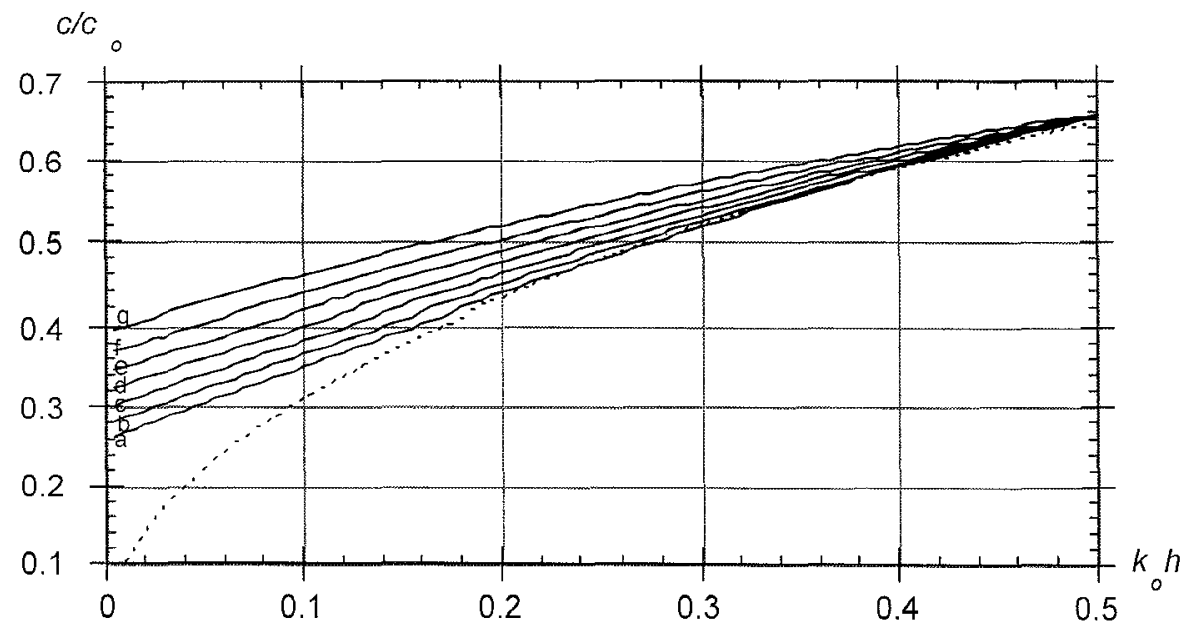

Figure $1:(-)$ Equation de dispersion non-linéaire (3) (Grilli, 1998) avec $k_{0} H_{0}=0.02-0.14$ (a-g); (- - ) Equation de dispersion linéaire (1).

Figure $1:(\longrightarrow$ Nonlinear wave dispersion relationship (3) (Grilli, 1998) with $k_{0} H_{0}=0.02-0.14$ (a-g); (- -) linear wave dispersion relationship (1).

\section{Principe de l'inversion de la profondeur}

Grilli (1998) présente des calculs en canal à houle numérique, basés sur les équations d'un fluide potentiel avec équations de surface libre totalement nonlinéaires, appliqués au shoaling de vagues périodiques sur fonds de pentes faibles. Ces résultats permettent d'établir, sur la base d'ajustements empiriques aux résultats numériques, la relation de dispersion (Fig. 1),

$$
c / c_{0}=A_{0}+A_{1}\left(k_{0} h\right)+A_{2}\left(k_{0} H_{0}\right)+A_{3}\left(k_{0} h\right)\left(k_{0} H_{0}\right)+A_{4}\left(k_{0} h\right)^{2}+A_{5}\left(k_{0} H_{0}\right)^{2}(3)
$$


Comme attendu, on voit que les vitesses de phases non-linéaires normalisées $c / c_{0}$ sont plus grandes que celles prédites par la théorie linéaire, et d'autant plus que la cambrure des vagues incidentes augmente, à cause de la dispersion d'amplitude.

Les variations des hauteurs de vagues calculées durant le shoaling sont présentées à la Fig. 2, où l'on voit que la paramétrisation sélectionnée, $H c_{0} /\left(H_{0} c\right)$, permet de superposer toute les courbes, avant que le point de déferlement ne soit atteint. On voit également,ce qui est surprenant, que la solution linéaire prédit cette paramétrisation assez précisément. [Les non-linéarités de $H$ et $c$ se compensant à peu près, les cambrures calculées par la théorie linéaire et non-linéaire deviennent presque identiques.] La combinaison de ces résultats avec (3) permet donc de représenter $H$ numériquement et, si l'on dispose de hauteurs de vagues mesurées, de prédire $k_{0} H_{0}$, ce qui est nécessaire à la prédiction de la célérité, et à l'inversion non-linéaire de profondeur.

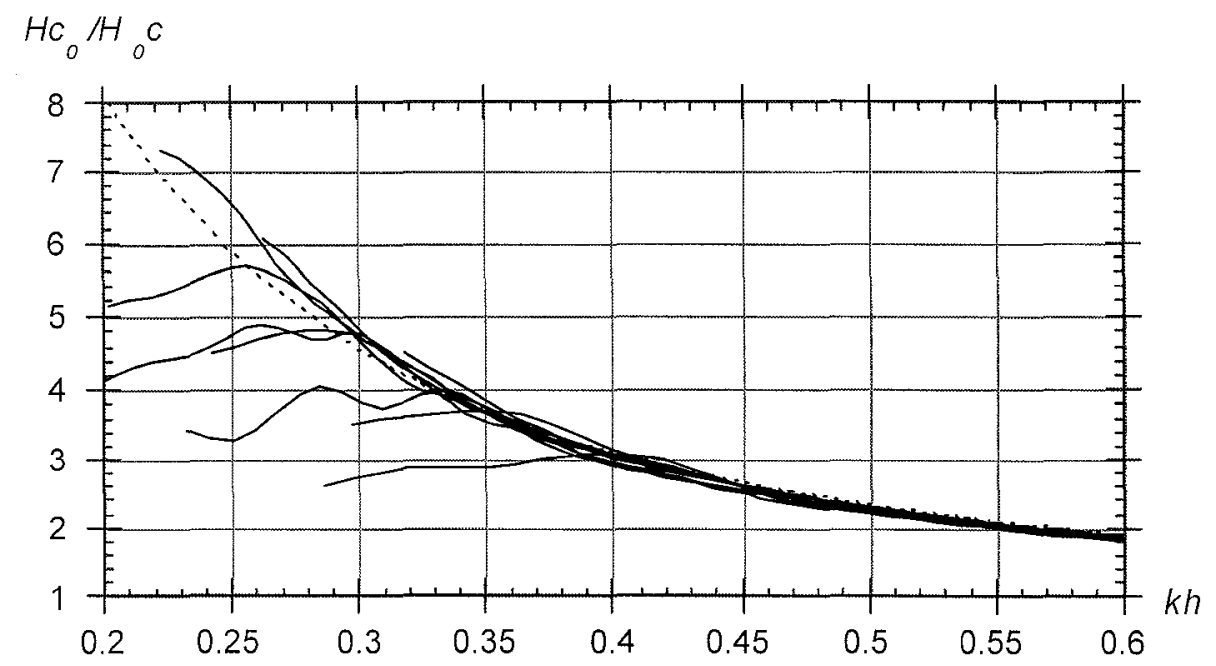

Figure 2: $\left(\rightarrow\right.$ ) Vagues non-linéaires (Grilli, 1998) avec $k_{0} H_{0}=0.02-0.14$; (- - ) Solution du shoaling linéaire.

Figure 2: $\rightarrow$ Nonlinear waves (Grilli, 1998), with $k_{0} H_{0}=0.02-0.14$; (- - ) linear shoaling solution

Nous verrons que les données de terrain disponibles par télédétection ne fournissent pas aisément et/ou précisément les hauteurs des vagues. Pour appliquer notre procédure d'inversion, un nouvel algorithme est développé dans lequel ces hauteurs sont estimées en utilisant la Fig. 2, sur la base de la hauteur des vagues au point de déferlement $H_{b}$. Plus exactement, une première inversion de profondeur $h(x)$ est effectuée sur la base de l'équation de dispersion linéaire (1), de données de célérité $\left(c\left(x_{i}\right) ; i=1, \ldots, \mathrm{N}\right)$ et de période moyenne $T_{m}$ pour les vagues incidentes, ainsi que de la distance $x_{b}$ à la plage, mesurée à partir du point de déferlement (aisément observable sur des photos aériennes comme le début de la zone d'écume blanche; Fig. 3). Un critère de déferlement est ensuite utilisé pour estimer $H_{b}$ sur la base de la profondeur $h_{b}$ au point de déferlement, obtenue en fonction de $x_{b}$ et de $h(x)$,

$$
H_{b}=\gamma\left[k_{0} H_{b}, m\right] h_{b}
$$


où $m$ est la pente au point de déferlement et $k_{0}=(2 \pi / T)^{2} / g$ est obtenu sur la base de la théorie linéaire. Sur la base de la Fig. 2 et de $\left(k h_{b}, H_{b}, c_{0}\right)$ on peut alors obtenir $k_{0} H_{0}$ et utiliser (3) pour effectuer une inversion de profondeur non-linéaire. Le processus est répété itérativement jusqu'à convergence des résultats.

Le reste de cette article discute du problème de l'obtention de données et des résultats obtenus à l'aide de celles-ci.

\section{Traitement préliminaire des données de luminance}

La Figure 3 présente un exemple d'image produite par le système de télédétection optique AROSS (Dugan et al., 2001), lors de la campagne SHOWEX (SHOaling Wave Experiment) à Duck, N.C., USA. Ce système se compose d'une caméra digitale combinée à des appareils de localisation géographique de la zone survolée, embarqués à bord d'un petit avion.

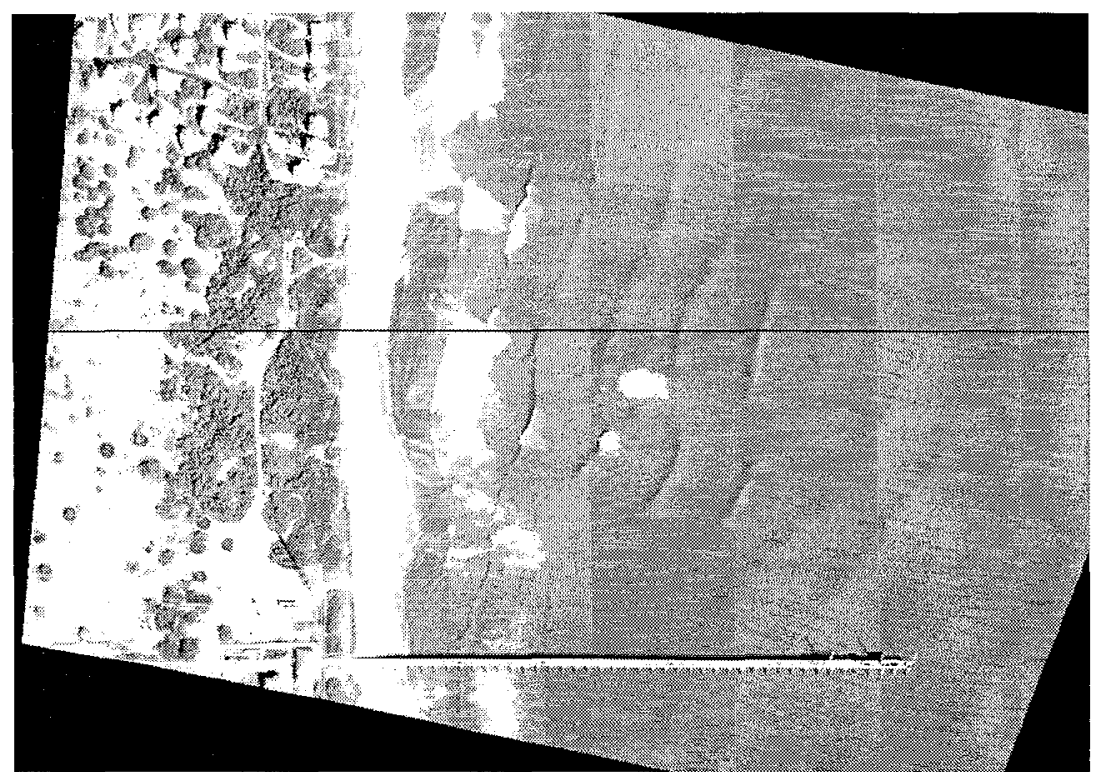

Figure 3. Exemple de photo instantanée de houle en shoaling prise par AROSS. La ligne noire représente un transect considéré.

Figure 3. AROSS snapshot showing brightness variations. Black line = transect along which depth is calculated.

Le premier traitement des images, telles que présentées à la Fig. 3 (Dugan et Williams, 2000), consiste à produire les variations dans le temps de la luminance associée aux pixels noirs et blancs (valeurs de 0 à 255), $b(x, t)$, le long du transect. Les images sont prises à 0.5 seconde d'intervalle et une bande de 5 mètres (dans la direction parallèle à la côte) entourant le transect étudié est moyennée afin de lisser quelque peu le signal de luminance. La Figure 4 montre le résultat de ces opérations pour une partie du domaine spatio-temporel. Les lignes claires 
représentent les maxima de luminance qui se produisent près des crêtes de vagues et permettent ainsi de localiser les trajectoires des crêtes $x_{c}(t)$, dont la pente temporelle fournit la vitesse de phase des vagues $c=\mathrm{d} x_{c} / \mathrm{d} t$. La zone des « eaux blanches » correspond à la zone de déferlement.

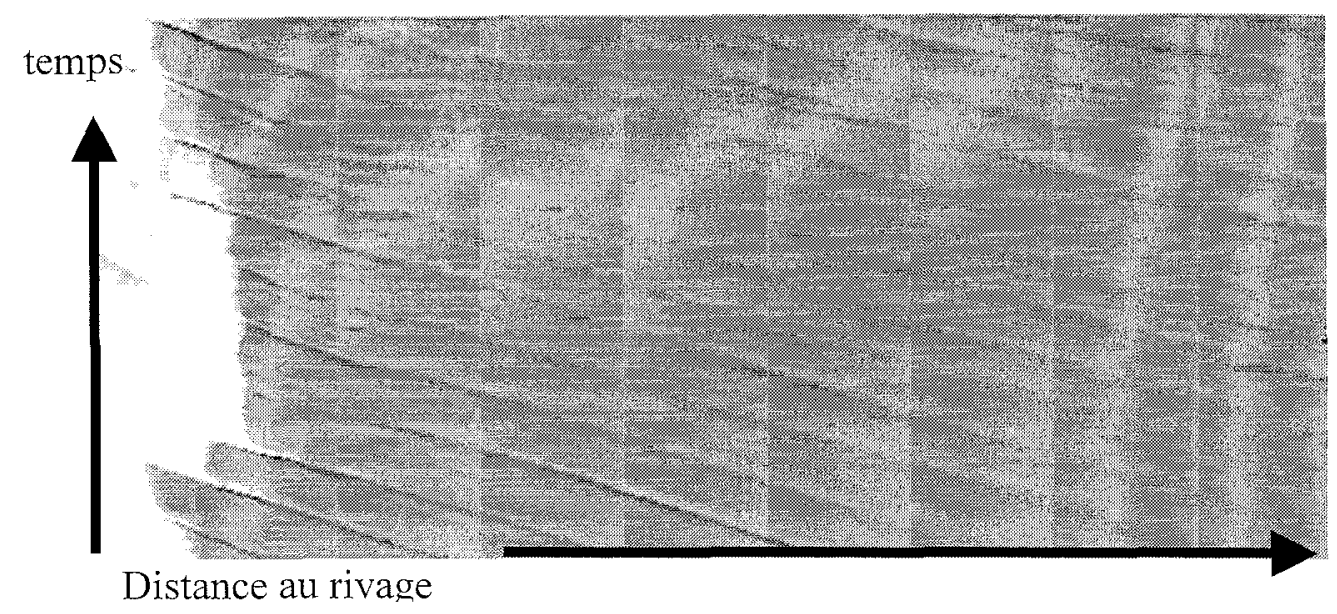

Figure 4 : Diagramme de luminance espace-temps le long du transect de la Fig. 3. Figure 4: Space-time brightness variations along Fig 3 transect

Les positions des maxima de luminance correspondent donc aux trajectoires des crêtes. L'algorithme ci-dessous décrit plus précisément le calcul automatique des trajectoires de crêtes :

1) L'image exploitable de la Fig. 4, extraite de Fig. 3 est transcrite en valeurs numériques de 0 (noir) à 255 (blanc). Cette image/matrice est analysée ligne par ligne, $c$-à-d, pour chaque temps, on s'intéresse aux variations de la luminance selon la position sur le transect : $b(x)$.

2) Pour chaque temps, la tendance de luminance $b_{0}(\mathrm{x})$ (obtenue par un polynôme du 3ème degré) est soustraite : $b^{\prime}=b$ - $b_{0}$. La matrice ainsi obtenue est la matrice des luminances corrigées de la tendance.

3) Les points de 'zero-up-crossing' (passage par zéro par valeurs croissantes ; ZUC) sont identifiés pour chaque ligne de $b^{\prime}$

4) Les maxima de $b^{\prime}$ sont identifiés entre les points de ZUC.

5) Les valeurs de $b^{\prime}$ max ainsi obtenues sont filtrées pour éliminer les maxima locaux, en utilisant divers critères tels qu'une valeur seuil de luminance caractérisant un maximum, et une distance minimum séparant deux maxima successifs.

6) Pour la matrice toute entière, il faut à présent identifier la position de chaque crête parmi les positions des maxima de luminance : $x_{c}=x\left(b^{\prime}{ }_{\text {max }}\right)$, en prenant soin d'éliminer des fausses crêtes (crêtes localisées artificiellement par l'algorithme).

La Figure 5 montre le résultat de la recherche automatique des trajectoires de crêtes à partir de l'algorithme ci-dessus appliqué à la Fig. 4 (l'axe des temps a été inversé pour obtenir des célérités positives). Le signal est relativement clair dans la zone proche du rivage, du fait de la cambrure marquée des vagues mais, dans 
cette zone, il y a formation d'harmoniques secondaires (nettement visibles sur l'image). Ceci rend plus difficile la distinction automatique entre vraies et fausses crêtes primaires de la houle, comme on peut le voir à la Fig. 5. Pour pallier cela, il est possible de procéder à une détection semi-automatique des crêtes en repérant manuellement la position de chaque crête à proximité du rivage et digitalisant leur trajectoire.

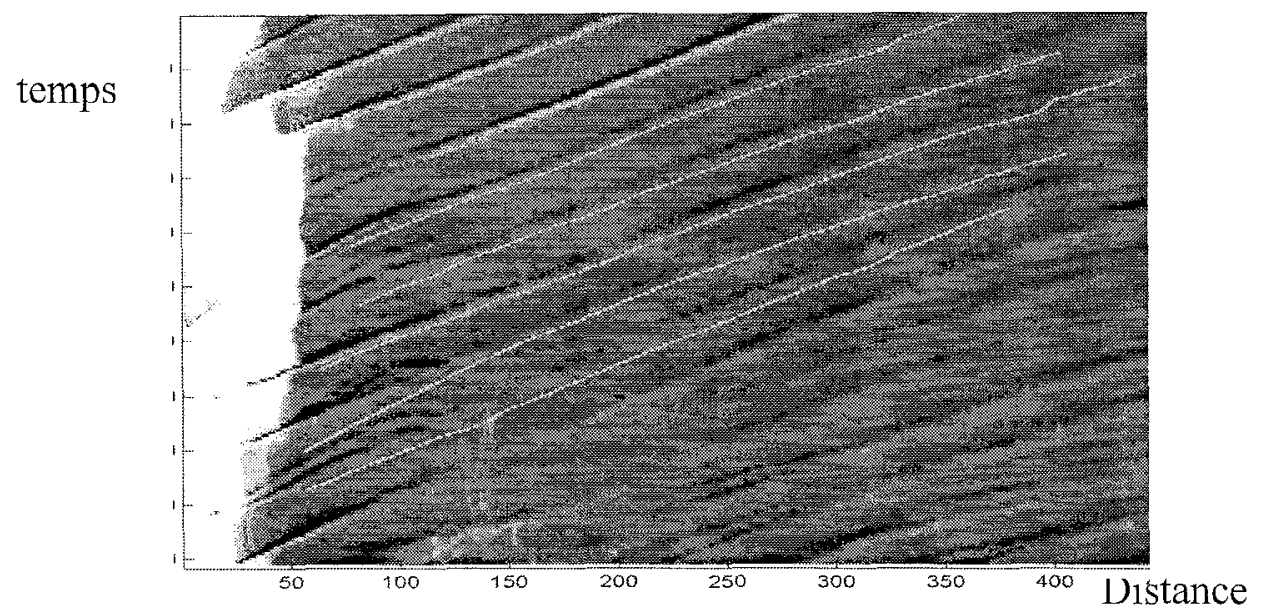

Figure 5. Résultats de la recherche automatique de crêtes pour la Fig, 4 Figure 5. Automatic detection of wave crest trajectories in Fig. 4

Les propriétés des vagues (vitesse de phase c et période $T$ ) sont calculées pour chaque trajectoire de crête reconstituée. La pente temporelle des trajectoires fournit la vitesse de phase. La distance entre crêtes donne la longueur d'onde $L(x)$. La période est calculée comme la moyenne de $L(x) / c$, pour une trajectoire donnée.

\section{Inversion de la profondeur}

La hauteur de vague normalisée $k_{0} H_{0}$ est nécessaire au calcul de la profondeur par la méthode d'inversion utilisée. Cependant, la fonction de transfert luminancehauteur de vague n'étant pas connue, les hauteurs de vague $H$ ne peuvent pas être déduites directement des images de luminance. Ce paramètre est estimé en se basant sur la position du point de déferlement comme expliqué plus haut.

Les propriétés des vagues étant à présent déterminées, la relation de dispersion peut être inversée. Ces calculs de profondeur sont répétés pour chaque crête puis moyennés. La Figure 6 montre les variations de la bathymétrie moyenne calculée par la relation de dispersion linéaire superposée aux bathymétries moyenne et minimum calculées par l'algorithme non-linéaire (à partir des données obtenues semi-automatiquement), ainsi qu'à la bathymétrie réelle. Cette bathymétrie réelle est donnée par la moyenne des profondeurs mesurées indépendamment pour des sites proches du transect 


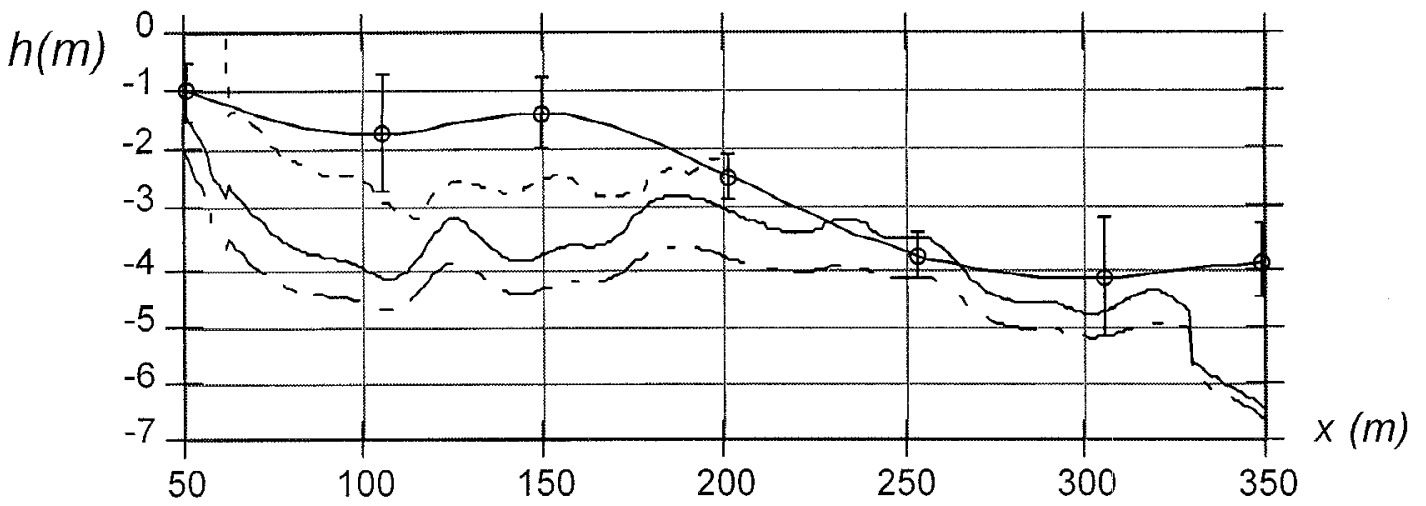

Figure 6. Profondeurs calculées d'après la relation linéaire (- -) et non linéaire moyenne (-) et minimum (---), superposées à la profondeur réelle moyennée autour du transect considéré $(-\mathrm{O}-)$.

Figure 6. Depth predicted by linear theory (-) and non linear theory (minimum (--) and mean (-)), superimposed to measured bathymetry (-O--).

\section{Conclusion}

Le nouvel algorithme d'inversion de profondeur, combiné aux données de terrain de luminance donne une bonne prédiction de la bathymétrie dans la zone de shoaling non-linéaire. Comme attendu, les profondeurs résultant de l'inversion non-linéaire, sont plus faibles que celle obtenues par la théorie linéaire (dispersion d'amplitude). En eau profonde, les résultats obtenus par les théories linéaire et non-linéaire convergent. Les profils de profondeurs obtenus présentent les mêmes caractéristiques générales que le profil de profondeur mesuré sur les 400 premiers mètres [Note l'axe des $x$ commence à 75 mètres]. Des améliorations sont à apporter à la détection automatique des crêtes afin d'éviter les bifurcations des trajectoires dues aux crêtes d' harmoniques secondaires. Ces calculs de bathymétrie devront également être réalisés pour un nombre plus important de crêtes et leurs résultats superposés ou moyennés afin d'obtenir les enveloppes ou moyennes des prédictions de bathymétrie. Enfin, il serait souhaitable de comparer les hauteurs de vague calculées aux hauteurs mesurées sur le terrain.

\section{Références}

Grillli, S.T., 1998, Depth inversion in shallow water based on nonlinear properties of shoaling periodic waves. Coastal Engng., 35(3), 185-209

Grilli, S.T. and J., Horrillo 1997, Fully nonlinear properties of periodic waves shoaling over mild slopes. In Proc. 25th Intl. Conf. on Coastal Engineering (ICCE25, Orlando, USA, September 96), Vol. 1, pps. 717-730. ASCE Edition.

Dugan, J.P et J.Z.Williams, 2000. Communication personnelle

Dugan, J.P., Piotrowski, C.C. and J.Z. Williams, 2001, Water depth and surface current retrievals from airborne optical measurements of surface gravity wave dispersion. J. Geophys. Res., 106(C8), 16,903-16,915. 